\title{
The disappearing hand: Vestibular stimulation does not improve hand localisation.
}

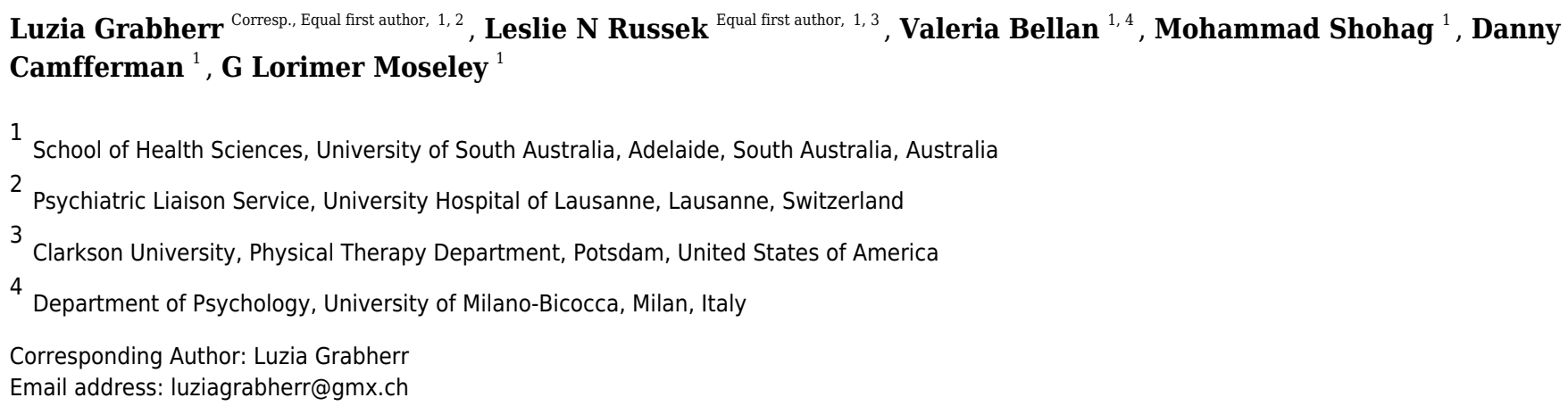

Background. Bodily self-consciousness depends on the coherent integration of sensory information. In addition to visual and somatosensory information processing, vestibular contributions have been proposed and investigated. Vestibular information seems especially important for self-location, but remains difficult to study.

Methods. This randomised controlled experiment used the MIRAGE multisensory illusion box to induce a conflict between the visually- and proprioceptively-encoded position of one hand . Over time, the perceived location of the hand slowly shifts, due to the fact that proprioceptive input is progressively weighted more heavily than the visual input. We hypothesised that left cold caloric vestibular stimulation (CVS) augments this shift in hand localisation.

Results. The results from 24 healthy participants do not support our hypothesis: CVS had no effect on the estimations with which the perceived position of the hand shifted from the visually- to the proprioceptively-encoded position. Participants were more likely to report that their hand was 'no longer there' after CVS. Taken together, neither the physical nor the subjective data provide evidence for vestibular enhanced self-location. 


\section{The disappearing hand: Vestibular stimulation does} 3 not improve hand localisation.

6 Luzia Grabherr ${ }^{1,2^{*}}$, Leslie N. Russek ${ }^{1,3^{*}}$, Valeria Bellan ${ }^{1,4}$, Mohammad Shohag ${ }^{1}$, Danny

7 Camfferman $^{1}$ and G. Lorimer Moseley ${ }^{1}$

$9{ }^{1}$ School of Health Sciences, University of South Australia, Adelaide, Australia

102 Psychiatric Liaison Service, University Hospital of Lausanne, Lausanne, Switzerland

113 Physical Therapy Department, Clarkson University, Potsdam, USA

$12{ }^{4}$ Department of Psychology, University of Milano-Bicocca, Milan, Italy

*These authors contributed equally

Corresponding Author:

17 Luzia Grabherr ${ }^{1}$

18 Psychiatric Liaison Service, University Hospital of Lausanne, Av. de Beaumont 23, 1011

19 Lausanne, Switzerland

Email address: Luzia.Grabherr@chuv.ch 


\section{Abstract}

24 Background. Bodily self-consciousness depends on the coherent integration of sensory information. In addition to visual and somatosensory information processing, vestibular 26 contributions have been proposed and investigated. Vestibular information seems especially 27 important for self-location, but remains difficult to study.

28 Methods. This randomised controlled experiment used the MIRAGE multisensory illusion box 29 to induce a conflict between the visually- and proprioceptively-encoded position of one hand. 30 Over time, the perceived location of the hand slowly shifts, due to the fact that proprioceptive 31 input is progressively weighted more heavily than the visual input. We hypothesised that left 32 cold caloric vestibular stimulation (CVS) augments this shift in hand localisation.

33 Results. The results from 24 healthy participants do not support our hypothesis: CVS had no 34 effect on the estimations with which the perceived position of the hand shifted from the visually35 to the proprioceptively-encoded position. Participants were more likely to report that their hand 36 was 'no longer there' after CVS. Taken together, neither the physical nor the subjective data 37 provide evidence for vestibular enhanced self-location. 


\section{Introduction}

The important contribution of accurate and congruent sensory information to bodily selfconsciousness has been widely acknowledged (e.g. Blanke, 2012; Blanke \& Metzinger, 2009; Serino et al., 2013). Besides visual and somatosensory information, vestibular contributions have also been suggested and investigated. Particularly, research in patients involving altered bodily awareness (e.g. neglect, somatoparaphrenia, phantom limb sensations) suggests that vestibular stimulation can attenuate dysfunctional bodily self-consciousness (for reviews see e.g. Grabherr, Macauda, \& Lenggenhager, 2015; Lopez, 2015; Mast, Preuss, Hartmann, \& Grabherr, 2014). Patients with impaired vestibular function on the other hand are prone to distorted own body representations (Lopez et al., 2018) and out-of-body experiences (Lopez \& Elziere, 2018).

Bodily self-consciousness is thought to consist of at least three different components: body ownership (the experience of owning a body), first-person perspective (the experience of taking a visuospatial perspective from that body), and self-location (the experience of being a body in a given location) (Blanke \& Metzinger, 2009; Serino et al., 2013). Recently, Serino and colleagues (2013) have proposed that bodily ownership and self-location rely on partially distinct neural correlates, i.e. the premotor cortex and the temporo-parietal junction, respectively. The temporoparietal junction is likely a key player in the integration of vestibular and other sensory cues (Ionta et al., 2011; Pfeiffer, van Elk, Bernasconi, \& Blanke, 2016; see also Gallace \& Bellan, 2018). Given that the vestibular system provides important information about body position and self-motion in space, it has been proposed that vestibular information is particularly relevant to self-location (Lenggenhager \& Lopez, 2015; Pfeiffer et al., 2013; Serino et al., 2013).

Bodily illusions provide clever experimental tools because they can disrupt different components of bodily self-consciousness also in healthy participants. For example, during the rubber hand illusion (RHI) (Botvinick \& Cohen, 1998), participants are asked to rest their hands on a table. One of their hands is hidden and 'replaced' by a prosthetic (rubber) hand. The experimenter then starts stroking the rubber hand and the participants' hidden hand synchronously. After a few seconds, participants start to perceive that the rubber hand belongs to them. In addition, when asked to localise their hidden hand, they point at a spot between the real and the rubber hand. This localisation error is called "proprioceptive drift" and it has been correlated with the strength of the illusion (e.g. Botvinick \& Cohen, 1998; Tsakiris \& Haggard, 2005; but also see, for example, Rohde, Di Luca \& Ernst, 2011). The MIRAGE multisensory illusion box allows the manipulation of self-location of a body part specifically by using a compelling perceptual illusion called the disappearing hand trick (DHT) (Newport \& Gilpin, 2011). Participants see a video in real-time of their hands moving inwards, while, in fact their hands are moving outwards. Therefore, when the hands are placed on the bottom of the MIRAGE box, in line with the visual input, participants believe that their hands are close together, while they are in fact far apart. This is due to an induced sensory mismatch of up to 14 $\mathrm{cm}$, between the proprioceptively-encoded location of the participants' hidden hand (where the participants feel the hand to be), and its visually-encoded location (where the participants saw their hand for the last time), while participants remain unaware of this mismatch (Bellan et al., 
2017a; Bellan et al., 2015) (see Fig. 1). Recent research shows that self-location of the hand drifts over time from the last seen location towards the actual physical location, without the participants realising. This sensory mismatch allows testing of the relative weighting of visual and proprioceptive information in generating the perceived location of the hand, while minimising confounding effects introduced by expectation or report bias (Bellan et al., 2017a; Bellan et al., 2015).

Please insert Fig. 1 around here

Thus far, studies in healthy participants have suggested that caloric vestibular stimulation (CVS, please refer to the method section "caloric vestibular stimulation" for more information on this technique) and in particular left cold CVS (predominantly activating the right hemisphere) can enhance somatosensory perception (i.e. tactile perception) in the left and right hand (Ferrè et al., 2011; Ferrè et al., 2013). Another study using left anodal and right cathodal galvanic vestibular stimulation (GVS, which also predominantly activates the right hemisphere) reported reduced mislocalisation of one's own hand (i.e. enhanced proprioception) in the RHI (Ferrè, Berlot, \& Haggard, 2015) leading to the proposition that "vestibular signals rebalance sensory events away from the world and towards the self" (Ferrè \& Haggard, 2016, p. 77). This implies that vestibular stimulation may enhance weighting of proprioceptive information during the sensory mismatch induced via MIRAGE.

However, given the strong interrelation of visual and vestibular signals, some factors should be accounted for. First, it is also plausible that vestibular stimulation would enhance the weighting of visual information. Relevant to this possibility are results from other RHI experiments, suggesting that vestibular stimulation enhances visual capture and not proprioceptive information. Ponzo et al. (2018) found an enhanced proprioceptive drift towards the rubber hand. Although Lopez et al. (2010) found no effect of vestibular stimulation on the proprioceptive drift, they did find increased illusory ownership of the rubber hand and location of touch evidenced by questionnaire responses. In a subsequent experiment using a non-visual variant of the RHI, vestibular stimulation affected neither subjective feelings of body ownership nor proprioceptive drift, suggesting no influence of vestibular stimulation when visual feedback is absent (Lopez et al., 2012). If this were the case (i.e., that vestibular stimulation affects only on visual capture), we would observe a slower drift and/or a starting location that is closer to the visual hand location, when compared to a sham condition. Second, vestibular stimulationinduced attention effects have been debated. Potential vestibular attentional effects are typically observed towards the ipsilateral side of the stimulated ear. Thus, left cold CVS (as used in our study) could potentially lead to a generic attentional shift towards the left hemispace. However, such effects are critically debated and even when they are observed, they are usually short-lived in healthy participants; therefore, we did not predict such effects (see Bottini et al., 2013, for a critical discussion). In order to rule out such effects, we tested both hands, because they would be affected differently (the initial location and/or drift of the right hand would be shifted toward 
119 the proprioceptively-encoded hand position, whereas the initial location and/or drift of the left

120

121

122

123

124

125

126

127

128

129

130

131

132

133

134

135

136

137

138

139

140

141

142

143

144

145

146

147

148

149

150

151

152

153

154

155

156

157

hand would be shifted toward the visually-encoded hand position).

Clarifying the potential role of the vestibular system in bodily self-consciousness is important because vestibular stimulation has been proposed for treating a wide range of conditions involving body awareness, despite a lack of strong supportive evidence (for critical reviews see e.g. Grabherr et al., 2015; Miller, 2016). Findings from this study may help further the understanding of the role of vestibular information in self-location, particularly regarding the prioritisation of conflicting sensory input (i.e. visual vs. proprioceptive). This knowledge may help explain underlying mechanisms responsible for observed vestibular induced effects in patients with impaired bodily self-consciousness.

Taken together and despite some contradictory findings, we hypothesised that left cold CVS augments the self-location component of bodily self-consciousness. This should be reflected by a more pronounced shift from visual to proprioceptive location in the MIRAGE illusion, such that vestibular stimulation compared to a sham condition leads to (i) a faster drift in perceived location, toward the proprioceptively-encoded hand position, (ii) an initial perceived location that is closer to the proprioceptively-encoded hand position, or (iii) both.

\section{Materials \& Methods}

\section{Participants}

An a priori power analysis was performed to determine the number of participants. Given the use of a novel tool to assess hand localisation after CVS, it was difficult to calculate effect sizes based on previous data. Therefore, we assumed a generic medium to large effect size of $f=$ 0.3 with a power $=0.8$. Estimations were performed with MorePower 6.0 (Campbell \& Thompson, 2012) detecting a required sample of 22 participants. We recruited 24 to allow for a 2 $\mathrm{x} 2$ counterbalanced design. This sample size is slightly bigger than previous studies testing the influence of vestibular stimulation on bodily self-consciousness and body representation but using different tasks (Lopez et al., 2010; Lopez, Schreyer, Preuss, \& Mast, 2012). Participants were recruited through noticeboards and social media. The mean age was $25 \pm 9 \mathrm{SD} ; 14$ were female. One participant was replaced because no CVS response could be evoked (no nystagmus and no reported subjective effects). All participants needed to be right-handed according to a shortened version of the Edinburgh Handedness Inventory (Veale, 2014) and had normal or corrected-to-normal vision. Participants were assessed with a short medical history questionnaire and were excluded if they reported otological, neurological, or psychological problems. A physician verified the integrity of the eardrum (otoscopy) before and after the experiment. Participants received financial compensation for their participation.

The study was approved by the local ethics committee (University of South Australia, Application ID: 32955) and was performed in accordance with the Declaration of Helsinki. Written informed consent was obtained before commencing the experiment. 
158

159

160

161

162

163

164

165

166

167

168

169

170

171

172

173

174

175

176

177

178

179

180

181

182

183

184

185

186

187

188

189

190

191

192

193

194

195

196

197

\section{Caloric vestibular stimulation}

Caloric vestibular stimulation (CVS) was performed with participants lying supine with their head positioned $30^{\circ}$ upright, in order to place the horizontal semicircular canal in a vertical position. The left external ear canal was irrigated for 2 minutes with $100 \mathrm{ml}$ of cold water $\left(20^{\circ}\right.$ C). Typically, left cold CVS elicits a slow-phase nystagmus towards the left side and a fast-phase nystagmus towards the right side accompanied by an illusory feeling of self-motion to the right and predominantly activates the right hemisphere, where the left hand is represented (Lopez, Blanke, \& Mast, 2012). Importantly, the right hemisphere is the dominant hemisphere for vestibular function (Dieterich et al., 2003). Sham stimulation consisting of irrigating the left ear with water at body temperature $\left(37^{\circ}\right)$ was used as a control. The sham stimulation elicits similar extra-vestibular cues (e.g. tactile stimulation), but is widely viewed as not leading to an activation of the vestibular system (Miller \& Ngo, 2007). Two experimenters verified the effectiveness of the stimulation by visually assessing the nystagmus. A vestibular stimulation questionnaire (adapted from Lenggenhager, Lopez, \& Blanke, 2008; Lopez et al., 2010) assessed the subjective effects of vestibular stimulation. In particular, illusory motion was assessed on a numerical rating scale from 0 to $6(0=$ no motion, $6=$ very strong motion $) .13$ other common symptoms associated with vestibular stimulation were also assessed on a numerical rating scale from 0 to $6(0=$ absent, $6=$ severe $)$. These were general discomfort, nausea, vertigo, racing heartbeat or palpitations, difficulty concentrating, drowsiness, faintness, sweating or cold sweat, need to vomit, headache, fatigue, pallor, and blurred vision (please see Supplementary material, Fig. S2a and b). The vestibular stimulation questionnaire was administered before (in order to familiarise participants with the questionnaire and to clarify any clinical terms), and immediately after CVS and sham stimulation.

In line with other experimental protocols, adaptation and self-location testing with the MIRAGE system (see below) were conducted after the nystagmus had subsided, in order to prevent perceptual biases induced by the directional beating of the nystagmus itself. This is possible since CVS studies have revealed effects that outlast the duration of the stimulation as shown by several neuroimaging and behavioural studies (please see Ferrè, Haggard, Bottini \& Iannetti, 2015, for a thorough discussion). Participants were able to rest for 30 minutes between the two conditions, to minimise a possible carry-over effect.

\section{Apparatus (MIRAGE multisensory illusion box) and experimental setup}

The MIRAGE system consists of a 2-chamber box with a double sided mirror between the bottom chamber and the top chamber, and a video screen facing down placed on the ceiling of the top chamber. The system is arranged so that video images of the lower chamber are projected onto the mirror of the top chamber. Therefore, the mirror appears to show what is in the chamber below. The result is that, when the participants look down towards their hands, they see an image of their own hands at approximately the same location as the actual hands. Participants comfortably sit in front of the box while a bib blocks the view of their arms or any other visual 
198

199

200

201

202

203

204

205

206

207

208

209

210

211

212

213

214

215

216

217

218

219

220

221

222

223

224

225

226

227

228

229

230

231

232

233

234

235

236

237

cues about their hand position. Using customised software, the projected image is capable of being manipulated in order to create the visual illusion that the participants' hands were closer together than they actually were, thus making the visual and proprioceptive input incongruent (see Fig. 1). For more details on the MIRAGE, see https://miragelab.co.uk/.

\section{Experimental procedure}

Participants first learned and practised a well-established localisation task (Bellan et al., 2015) with accurate visual information. They placed their hands into the MIRAGE box with a computer-generated red arrow projected at the midpoint between the hands while seeing an image of the hands at their actual location. The arrow moved towards the tested hand at a constant velocity (approximately $2.86 \mathrm{~cm} / \mathrm{sec}$ ) and participants said "stop" when the arrow was pointing at the image of their middle finger. Participants practised this localisation task at least 6 times while able to see an image of the tested hand in the MIRAGE box, or until participants were comfortable with their accuracy in performing the task. The tested hand was then hidden from view and participants repeated the localisation task by saying "stop" when the arrow aligned with where they believed their middle finger to be; this was practised at least 5 times, or until participants were comfortable with the task. Throughout the experiment, the arrow always moved from the centre of the screen towards the unseen hand. The direction of movement of the arrow does not affect localisation judgments (Bellan et al., 2015). The time between practice trials was 15 seconds. After localisation practice for each hand, participants removed their hands from the box and completed a 7-item visual-analogue scale (VAS) questionnaire about their perceptions of the hand that was tested (see first column in Table 1). This provided an opportunity to clarify items on the questionnaire and familiarise participants with the process. Questions one and five were designed to assess self-location. This questionnaire was a shortened VAS version of that used in the original DHT experiment (Newport \& Gilpin, 2011). We used VAS over NRS (numerical rating scale) because of its superior psychometric properties (e.g. Price, Staud, \& Robinson, 2012).

During the actual experiment, the caloric or sham stimulation was performed as described above and, once nystagmus subsided, the participant walked to another, adjacent room where the localisation task was performed by a third experimenter, blinded to the condition (CVS or sham). Participants were instructed not to discuss or report symptoms to this experimenter and there was no conversation other than MIRAGE instructions and participant responses. First, the MIRAGE adaptation procedure was performed by having participants place their hands into the MIRAGE box, holding the hands approximately $5 \mathrm{~cm}$ above the floor of the box and not touching any portion of the box with their hands or forearms. During the adaptation procedure, participants were asked to keep their hands between two visible blue bars surrounding each hand. The visual image of the hands was manipulated so that the hands looked like they were gradually moving inwards. However, in order to keep the hands between the blue bars, participants were actually moving their hands further apart. At the end of the adaptation procedure, which lasted 25 seconds, the physical location of each hand was 150 pixels (approximately $13.65 \mathrm{~cm}$ ) lateral to 
238 the visual image of the hand (see Fig. 1). The experimenter then placed the participants' hands

239

240

241

242

243

244

245

246

247

248

249

250

251

252

253

254

255

256

257

258

259

260

261

262

263

264

265

266

267

268

269

270

271

272

273

274

275

276

277

on the floor of the box, where the hands remained until the first round of testing was complete.

Participants then performed the localisation task, by saying "stop" when the arrow aligned with where they believed their middle finger to be. The localisation task was performed 6 times at 15 -second intervals, and the stop position of the arrow recorded for each trial. After approximately every third trial, participants were verbally reminded to focus on where they felt their middle finger to be. The actual physical location of the middle finger was recorded by the experimenter. Participants then removed their hands from the MIRAGE box and completed the questionnaire about subjective experience of the hand that was just tested. This process was then repeated for the other hand. It is not known exactly how long the effects of CVS last, but prior research using CVS to modulate bodily sensations found changes that persisted for at least 15 minutes after the CVS (Ferré et al., 2013). Consequently, the MIRAGE and localisation tasks were performed as quickly as possible after the caloric or sham stimulation and always within this 15 -minute window. Testing of both hands was completed in an average of 10 minutes 36 seconds ( \pm 54 seconds) after completion of the CVS or sham; the maximum time required for MIRAGE testing was 14 minutes from completion of the CVS or sham. Hand (right vs. left) and condition (CVS vs. sham) were counterbalanced and randomised in blocks of four. After the first session, participants were able to rest for 30 minutes.

Finally, at the end of the experiment, participants were asked five debriefing questions to determine subjects' awareness of the illusion: 1 . What do you think we were studying in the experiment? 2. Did you notice a difference between the two stimulation conditions? 3. What do you think was happening in the position tracking part (i.e. when the hands were between the two blue bars) of the experiment? 4 . Were you aware that you were moving your hands during the position tracking part of the experiment? 5. If yes to previous question: In what direction were your hands moving?

\section{Statistical analysis}

Hand location error was calculated as the difference between the participants' estimated position and the last seen position of the middle finger. Last seen position was used rather than the physical position because we were interested in initial localisation and speed of drift. To test this, a linear regression was performed on location at the 6 time points, resulting in an intercept (reflecting initial displacement relative to visual location) and slope (reflecting speed of drift from visual to physical or proprioceptive location) for each hand and condition. Slope and intercept were analysed using a repeated measures analysis of variance (ANOVA) with the factors Stimulation (CVS, sham) and Hand (left hand, right hand) as within-subjects factors. Responses to many of the MIRAGE questions were not normally distributed (and could not be transformed to be normal) and were therefore analysed non-parametrically using Friedman TwoWay ANOVA by Ranks, with Stimulation (CVS, sham) and Hand (left hand, right hand) as the 4 conditions. Post-hoc analysis was done using Wilcoxon Signed Rank Test for CVS versus sham stimulation; Bonferroni correction was performed for multiple post-hoc tests $(p=.05 / 2$, as right

Peer) reviewing PDF | (2018:12:33926:1:2:NEW 10 May 2019) 
278

279

280

281

282

283

284

285

286

287

288

289

290

291

292

293

294

295

296

297

298

299

300

301

302

303

304

305

306

307

308

309

310

311

312

313

314

315

316

and left hands were analysed separately). Wilcoxon Signed Rank Test was also used to analyse the vestibular stimulation questionnaire (CVS versus sham). Statistical tests were performed with IBM SPSS Statistics 22.

To quantify how much the data should shift our belief in favor of the null or the alternative hypothesis, we computed Bayes Factors for the main analyses $\left(\mathrm{BF}_{10}\right.$ where 1 means that the two hypotheses are equally likely, larger values indicate more evidence for the alternative hypothesis, and smaller values indicate more evidence for the null hypothesis; $\mathrm{BF}_{01}$ where values above 3 indicate substantial evidence for the null hypothesis). All Bayesian analyses were computed with JASP version 0.8.6 using Cauchy-distributed objective priors, centered on zero, with a default scale of 0.707 (i.e. $50 \%$ of possible standardized effect size values are expected to fall between 0.707 and +0.707$)($ JASP Team, 2018).

\section{Results}

\section{Hand localisation}

As expected, localisation drifted from the last seen position outwards (i.e. where the hand actually was). Fig. 2 illustrates the average location error for each of the four conditions separately. However, as indicated by the ANOVA for slope, this error did not significantly differ between the four conditions: there was no significant main effect of Stimulation $(F(1,23)=$ $\left.0.036, p=.852, \eta_{p}{ }^{2}=.002, \mathrm{BF}_{10}=0.213, \mathrm{BF}_{01}=4.694\right)$, no significant main effect of Hand $(F$ $\left.(1,23)=1.016, p=.324, \eta_{p}{ }^{2}=.042, \mathrm{BF}_{10}=0.399, \mathrm{BF}_{01}=2.504\right)$, and no significant interaction Stimulation $\times$ Hand $\left(F(1,23)=0.283, p=.600, \eta_{p}{ }^{2}=.012, \mathrm{BF}_{10}=0.026, \mathrm{BF}_{01}=37.960\right)$.

The ANOVA for intercept also revealed no significant differences: there was no significant main effect of Stimulation $\left(F(1,23)=1.085, p=.308, \eta_{p}{ }^{2}=.045, \mathrm{BF}_{10}=0.284, \mathrm{BF}_{01}=3.521\right)$, no significant main effect of Hand $\left(F(1,23)=1.601, p=.218, \eta_{p}^{2}=.065, \mathrm{BF}_{10}=0.643, \mathrm{BF}_{01}=\right.$ $1.556)$, and no significant interaction Stimulation $\times$ Hand $\left(F(1,23)=2.012, p=.170, \eta_{p}{ }^{2}=.080\right.$, $\left.\mathrm{BF}_{10}=0.094, \mathrm{BF}_{01}=10.659\right)$.

Please note that the Bayes Factors support the null hypothesis.

Please insert Fig. 2 around here

\section{Questionnaire / Subjective reports of the MIRAGE}

Hypothesis testing (Stimulation and Hand) showed that Question 4 ("It seemed like my right/left hand was no longer there") was significant $(p=.036)$, with post hoc Wilcoxon tests for CVS vs. sham significant for the left hand $(p=.012)$ but not the right hand $(p=.039)$. None of the other questions revealed a significant difference between CVS and sham. See Table 1 for the statements of all questions and full statistical indices and please refer to Supplementary material Fig. S1 illustrating the results of the questionnaire. 
317

318

319

320

321

322

323

324

325

326

327

328

329

330

331

332

333

334

335

336

337

338

339

340

341

342

343

344

345

346

347

348

349

350

351

352

353

354

355

Please insert Table 1 around here

\section{Vestibular stimulation questionnaire}

\section{Illusory motion}

To assess illusory motion due to CVS, we asked participants: "Did you perceive any type of motion during the stimulation?" and, if they responded affirmative, they were questioned further about how strong this perception was for the whole body, upper body, head, arms, and, legs (please refer to the method section "caloric vestibular stimulation" for more details on the questionnaire). All items were different between conditions (all $p<.01$ ), indicating that participants - as expected - experienced stronger illusory motion perception after CVS than after sham. Ratings were highest for head motion (mean 3.9 $\pm 2.2 \mathrm{SD})$ and lower for the arms $(1.8 \pm$ 2.0 SD) and legs (1.4 $\pm 1.9 \mathrm{SD})$. One participant did not report experiencing illusory motion, but nystagmus was observed and other symptoms reported, so we were confident that CVS was effective in this participant. All other participants reported the strength of illusory motion to be 2 or greater in the CVS condition.

We also asked participants to describe in what direction they perceived themselves to move (perception of illusory own body-motion). While many participants reported experiencing a circular motion to the right, a few participants found it difficult to clearly indicate the directional quality or found it difficult to distinguish between illusory own-body motion and illusory motion of the room (i.e. visual field).

\section{Stimulation induced symptoms}

Despite some moderate general discomfort and other symptoms such as vertigo and dizziness, all participants were able to complete the experiment. One participant reported slight dizziness (between 1 and 2 on a scale from 0 - 6) up to five days later; while unusual, this long duration of effects was also observed once during a pilot test.

Participants frequently reported vestibular related side effects after CVS but seldom after sham (Supplementary material Fig. S2a and b). Most symptoms (10/13), namely, general discomfort, nausea, vertigo, racing heartbeat or palpitations, difficulty concentrating, drowsiness, faintness, sweating or cold sweat, need to vomit and blurred vision, were rated significantly higher (all $p<.05$ ) after CVS than after sham. Head tension or headache and fatigue were no more common after CVS than after sham $(p>.1)$; pallor was not significant $(p=.07)$. Most $(>50 \%)$ participants reported general discomfort and difficulty concentrating, $50 \%$ reported vertigo and fewer reported other symptoms (please refer to Supplementary material, Fig. S2a and b).

\section{Debriefing questions}


356

357

358

359

360

361

362

363

364

365

366

367

368

369

370

371

372

373

374

375

376

377

378

379

380

381

382

383

384

385

386

387

388

389

390

391

392

393

394

395

Participants were not aware of the precise hypothesis tested but they all noticed a difference between CVS and sham, typically pointing out that they experienced more and stronger side effects during CVS than they did during sham (see section above). Nine participants hinted that they believed that we were testing how CVS impairs their ability to perform the task.

Most participants reported that their hands moved as needed to remain between the two blue bars, but either said that they were moving only slightly back and forth, or inwards (i.e. towards each other). Four participants reported that their hands were in fact moving outwards (correct observation).

\section{Discussion}

We investigated whether caloric vestibular stimulation (CVS) changes the relative weighting of proprioceptive and visual information in self-location, or bodily perceptions of the hands, when participants receive conflicting visual and proprioceptive information regarding hand position. Our results show that, although the feeling of the target hand (i.e. the hand that was out of view and had to be localised) is different after CVS, as compared to after sham, there was no effect on perceived location of the hand, nor on the weighting of proprioceptive and visual information during self-location. These findings are evidenced by no differential effect of condition on the slope or intercept of the location data, but a stronger reported feeling after CVS that the hand was 'no longer there' (main effect question 4 of the MIRAGE questionnaire). Post hoc testing showed that this main effect seems to be driven by the results of the left hand (significant result for the left hand and a trend only for the right hand) possibly reflecting the predominant activation of the right hemisphere after left cold CVS and/or the right hemisphere dominance of the vestibular system.

Our results appear in line with some previous literature and in contrast to other. In particular, vestibular stimulation did not affect the objectively measurable change in self-location in a rubber hand illusion (RHI) paradigm (i.e. the proprioceptive drift) (Lopez, Bieri, et al., 2012; Lopez et al., 2010), but it did impact relevant subjective measures (i.e. increased illusory ownership of the rubber hand and the illusory location of touch) (Lopez et al., 2010). Our results extend our understanding of vestibular effects by showing that bodily feelings that are closely aligned with proprioception (the feeling of the hand being there) could change independently of proprioception. Our findings are in contrast however with previous results reporting that galvanic vestibular stimulation (GVS) can reduce the drift (i.e. reduce mislocalisation of one's own hand) in a RHI paradigm, showing enhanced weighting of proprioceptive input through vestibular stimulation (Ferrè et al., 2015). Our data diverge also with studies in healthy participants that showed impaired proprioception after GVS (Ponzo et al., 2018; Schmidt et al., 2013).

Participants were in general not aware of the mismatch between the seen and the physical position of the hand. For most participants, the MIRAGE illusion was effective in creating a mismatch between visual and proprioceptive localisation of the hands; only four participants were aware that their hands physically moved in a different direction from the visual image of

Peer) reviewing PDF | (2018:12:33926:1:2:NEW 10 May 2019) 
396

397

398

399

400

401

402

403

404

405

406

407

408

409

410

411

412

413

414

415

416

417

418

419

420

421

422

423

424

425

426

427

428

429

430

431

432

433

434

435

their hands. This is important because it overcomes a limitation of the RHI, where participants cognitively know the rubber hand is not part of them and that their own hand rests out of view. Moreover, doubt has been raised over the validity of proprioceptive drift as a 'biomarker' of embodiment of the rubber hand. Proprioceptive drift occurs after the asynchronous hand stroking condition, also when the illusion is not induced, and even when no hand is displayed at all (Rohde, Di Luca, \& Ernst, 2011). Data from modified versions of the MIRAGE multisensory illusion indirectly support the idea that proprioceptive drift observed during the RHI is more likely to reflect visual capture of touch than embodiment of the artificial limb (Bellan et al., 2017a; Bellan et al., 2015).

Participants in this study did not report perceptual changes that have been observed during the RHI after CVS, but did report perceptual changes reflecting absence or numbness of the hand. This raises the very important possibility that such perceptual reports reflect expectation bias. It is intuitively sensible that, when an illusion replaces ones' own hand with a rubber counterpart, the rubber hand might feel 'owned'. In contrast, when the current study removed the tested hand from view during localisation testing, participants reported that the hand "was no longer there" instead of lacking of ownership. This may simply reflect the importance of visual confirmation on body localisation.

A few participants in this study reported that they believed that we were testing how CVS impairs their ability to perform the task and no one expected it might improve their ability. It therefore seems crucial that participants were not aware of the task characteristics (mismatch in hand localisation). However, individual differences were considerable (as indicated by the large variance of localisation estimates) and future studies may benefit from identifying sources of inter-individual variability. Moreover, our results suggest that right-handers respond similarly to the mismatch in hand-location independent of whether their dominant or non-dominant hand disappeared. In fact, to our knowledge, this is the first report using the MIRAGE to assess selflocation bilaterally; we found right and left (dominant and non-dominant) hands had similar initial location relative to last observed visual location and similar drift from visual to proprioceptive (i.e. physical) location. Also, as predicted, we did not observe potential vestibular induced attentional effects as reflected by non-significant interactions between the two factors Stimulation and Hand. The corresponding Bayes Factors support the acceptance of the null hypothesis.

Differential effects on implicit (self-location) and explicit (what does the body part feel like) markers would not be predicted on the basis of the proposal (Moseley, Gallace, \& Spence, 2012) and clinical framework of the cortical body matrix (CBM) (Bellan, Wallwork, Gallace, Spence, \& Moseley, 2017b; Wallwork, Bellan, \& Moseley, 2017). The CBM proposes that a multimodal neural network subserves the regulation and protection of the body and the space around it, at both a physiological and perceptual level. A broad range of findings support the CBM idea: for example, limb-specific disruption of thermoregulation (Moseley et al., 2008) and altered histamine reactivity (Barnsley et al., 2011), induced by the RHI; efficacy of the RHI modulated by hand temperature (Kammers, Rose, \& Haggard, 2011); thermoregulation and RHI 
436 efficacy disrupted by TMS over multimodal posterior parietal cortex (Gallace, Soravia, Cattaneo, 437 Moseley, \& Vallar, 2014); spatially-defined disruption of thermoregulation, ownership, pain 438 (Moseley, Gallace, \& Iannetti, 2012), bodily perception (Reid et al., 2016) and motor 439 performance (Reid et al., 2017). The compelling nature of that research adds weight to our earlier

440

441

442

443

444

445

446

447

448

449

450

451

452

453

454

455

456

457

458

459

460

461

462

463

464

465

466

467

468

469

470

471

472

473

474 suggestion that the pattern of changes in how the body feels without changes in how the body is regulated potentially reflect reporting bias. Of course, this issue needs to be interrogated more fully, but we contend that null results in rigorous studies powered a priori, such as this one, are critical for this process. The Bayes Factors support the null hypothesis. These indices strengthen the idea that the null hypothesis may indeed be more likely than the alternative hypothesis, arguing against a falsely negative finding.

Although CVS does not improve self-location of a body part, it remains possible that it would improve full body location. While self-location is the term typically used in such contexts, it may be more accurate to describe 'body part location with respect to the self' (Blanke \& Metzinger, 2009; Lenggenhager \& Lopez, 2015). When using an embodied mental transformation task (often also referred to as egocentric mental rotation task), CVS appears to improve performance for whole bodies, but not for single body parts (i.e. hands) (Falconer \& Mast, 2012). Even though people with bilateral vestibular loss are worse than healthy participants at embodied mental transformations, the impairment is not affected by whether the whole body or body-parts are involved (Grabherr, Cuffel, Guyot, \& Mast, 2011; see also Wallwork, Butler, \& Moseley, 2013). Clearly more research is required. While technically more demanding to investigate full-body illusions compared to body-part illusions, the use of visuovestibular cue manipulation to induce and manipulate full-body illusions (Macauda et al., 2014; Preuss \& Ehrsson, 2018) appears a step in the right direction.

As pointed out in the introduction, research in patient populations suggests that vestibular stimulation can enhance bodily self-consciousness. For example, phantom limb pain decreases after CVS (Andre et al., 2001). The authors interpreted that finding by suggesting that CVS enhances both body awareness and phantom limb sensation (by replacing a deformed with a normal representation). The current study was not able to similarly enhance bodily selfconsciousness among healthy individuals. This may reflect a fundamental difference in vestibular effects between people with clinically impaired bodily self-consciousness and healthy controls. In fact, vestibular stimulation has been reported to enhance proprioceptive localisation (arm position sense) in patients with impaired bodily awareness (i.e. spatial neglect), but impair it in healthy controls (Schmidt, Keller, et al., 2013). A recent study investigating potential beneficial effects of CVS in patients with a body identity integrity disorder, however, reported a null result (Lenggenhager, Hilti, Palla, Macauda, \& Brugger, 2014), suggesting that not all conditions are benefitted by CVS. The background of possible publication bias and widespread promotion of vestibular stimulation benefits also points to the importance of publishing null results such as those found here (for a discussion see Grabherr et al., 2015; for other recent null results in healthy participants see Macrea et al., 2016 and patients see Deroualle et al., 2017). 
475 One potential limitation of the study is inadequate blinding of the experimenter collecting 476 MIRAGE data, even though we explicitly avoided any conversation regarding symptoms. While 477 this blinding may not have been sufficient in some cases due to possible observable effects 478 induced by CVS (e.g. pallor), error introduced by inadequate blinding would most likely have 479 increased differences in MIRAGE results as that was the hypothesis being tested. It is unlikely 480 that inadequate blinding would have incorrectly led to the negative result observed. Future

481

482

483

484

485

486

487

488

489

490

491

492

493

494

495

496

497

498

499

500

501

502

503

504

505

506

507

508

509

510

511

512

513 research should attempt to assess and further improve blinding, if possible.

Another potential limitation is the possible influence of head movements due to walking in-between stimulation and MIRAGE testing. Due to the required head tilt to deliver CVS, many studies have their participants' head and/or body position readjusted to an upright sitting position after CVS (e.g. Ferrè et al., 2013; Ferrè et al., 2013; Karnath et al., 2003; Ngo et al., 2007; Ngo et al., 2008) allowing to conduct the subsequent experimental condition in a more natural and to other studies comparable head and body position. Please note that typically during these experimental conditions, the participant's head is not restrained allowing head motion (see for example Karnath et al., 2003). Moreover, studies involving patients revealed long lasting CVS induced effects that must have "survived" the effects of walking (e.g. André et al., 2001; ArandaMoreno et al., 2019). Taken together, the results of these studies show that there are CVS induced effects that outlast primary effects such as nystagmus and that they are at least to a certain extent robust to head movements. However, a subtler or more specific impact of movements cannot be fully ruled out due to the vestibular system's inherent multisensory nature and we are not aware of a study precisely addressing this question (contrary to several studies addressing the question how vestibular stimulation affects walking). Yet, as outlined above, there is evidence that walking would not completely abolish these longer lasting CVS effects. For our protocol, we estimated that the advantage - the blinding of the experimenter without losing time (the MIRAGE experimenter was set-up and ready to go as soon as the participant came in) outweighed this potential disadvantage.

Galvanic vestibular stimulation (GVS) may be better suited than CVS to assess vestibular contributions to self-location because it can be applied in a more precise fashion (Palla \& Lenggenhager, 2014) although compelling progress has been made improving CVS devices (Black et al., 2016). Although we undertook pilot studies and based our protocol on the available literature in the field, it remains possible that our CVS procedure artificially induced a vestibular input that was too strong to observe a beneficial effect (i.e. the aversiveness of the stimulation may cancel out the beneficial effects). Vestibular stimulation is often induced in experimental studies, as in this one, in a dichotomous fashion: it is either provided (experimental condition) or not (control condition). We have argued elsewhere that future experiments may need to consider more fine-tuned parameters taking into account the complexity and highly nuanced functioning of the vestibular system (Grabherr et al., 2015). We used double-verification of nystagmus (visual observation by a physician and a vestibular specialist), but did not include an objective, quantifiable nystagmus measure. This approach may have left us unable to identify participants 
514 with late-onset of nystagmus or a weaker response, both of which could possibly affect our 515 results.

516 Our final consideration is that we did not lodge and lock our experimental protocol prior to 517 conducting this experiment. Doing so enhances the transparency of research and is now

518 recommended practice for observational and clinical designs in many fields and including this 519 step would have enhanced the confidence with which the reader could accept our results (e.g. 520 Lee et al., 2018).

521

522

\section{Conclusions}

523

524

This investigation of whether CVS changes the relative weighting of proprioceptive and

525 visual information in self-location, or bodily perceptions of the hands, showed that although the subjective feeling of the hands appeared to be disrupted by CVS, there is no effect on self-

526 location of the hand, nor on the weighting of proprioceptive and visual information during self-

527 location. The methodological advantages of the MIRAGE procedure, and the naivety of

528

529 participants to the illusory manipulation, add weight to the finding that CVS does not improve self-location of a single body part.

530

531

\section{Acknowledgements}

532

533

We would like to thank Bigna Lenggenhager for her valuable advice on the manuscript and 534

535

536

537

538

539

540

541

542

543

544

545

546

547

548

549

550

551

Cat Jones for the pictures used in Figure 1.

\section{References}

André J, Martinet N, Paysant J, Beis J, and Le Chapelain L. 2001. Temporary phantom limbs evoked by vestibular caloric stimulation in amputees. Cogn Behav Neurol 14:190-196.

Aranda-Moreno C, Jáuregui-Renaud K, Reyes-Espinosa J, Andrade-Galicia A, Bastida-Segura AE, González Carrazco LG. Stimulation of the semicircular canals or the utricles by clinical tests can modify the intensity of phantom limb pain. Front Neurol 2019 10:117. 10.3389/fneur.2019.00117.

Barnsley N, McAuley JH, Mohan R, Dey A, Thomas P, and Moseley GL. 2011. The rubber hand illusion increases histamine reactivity in the real arm. Curr Biol 21:R945-946. 10.1016/j.cub.2011.10.039.

Bellan V, Gilpin HR, Stanton TR, Dagsdottir LK, Gallace A, and Lorimer Moseley G. 2017a. Relative contributions of spatial weighting, explicit knowledge and proprioception to hand localisation during positional ambiguity. Exp Brain Res 235:447-455. 10.1007/s00221-016-4782-6.

Bellan V, Gilpin HR, Stanton TR, Newport R, Gallace A, and Moseley GL. 2015. Untangling visual and proprioceptive contributions to hand localisation over time. Exp Brain Res 233:1689-1701. 10.1007/s00221-015-4242-8. 
552 Bellan V, Wallwork SB, Gallace A, Spence C, and Moseley GL. 2017b. Integrating self-

553

554

555

556

557

558

559

560

561

562

563

564

565

566

567

568

569

570

571

572

573

574

575

576

577

578

579

580

581

582

583

584

585

586

587

588 localization, proprioception, pain, and performance. J Dance Med Sci 21:24-35. 10.12678/1089-313x.21.1.24.

Black RD, Rogers LL, Ade KK, Nicoletto HA, Adkins HD, and Laskowitz DT. 2016. Noninvasive neuromodulation using time-varying caloric vestibular stimulation. IEEE $J$ Transl Eng Health Med 4:1-10.

Blanke O. 2012. Multisensory brain mechanisms of bodily self-consciousness. Nat Rev Neurosci 13:556-571. 10.1038/nrn3292.

Blanke O, and Metzinger T. 2009. Full-body illusions and minimal phenomenal selfhood. Trends Cogn Sci 13:7-13.

Bottini G, Gandola M, Sedda A, and Ferrè ER. 2013. Caloric vestibular stimulation: interaction between somatosensory system and vestibular apparatus. Front Integr Neurosci 7:66. 10.3389/fnint.2013.00066.

Botvinick M, and Cohen J. 1998. Rubber hands 'feel' touch that eyes see. Nature 391(6669):756. $10.1038 / 35784$.

Campbell JI, and Thompson VA. 2012. MorePower 6.0 for ANOVA with relational confidence intervals and Bayesian analysis. Behav Res Methods 44:1255-1265.

Deroualle D, Toupet M, Van Nechel C, Duquesne U, Hautefort C, and Lopez C. 2017. Anchoring the self to the body in bilateral vestibular failure. PLoS One 12(1):e170488.

Dieterich M, Bense S, Lutz S, Drzezga A, Stephan T, Bartenstein P, and Brandt T. 2003. Dominance for vestibular cortical function in the non-dominant hemisphere. Cereb Cortex 13:994-1007.

Falconer CJ, and Mast FW. 2012. Balancing the mind: vestibular induced facilitation of egocentric mental transformations. Exp Psychol 59:332-339. 10.1027/16183169/a000161.

Ferrè ER, and Haggard P. 2016. The vestibular body: Vestibular contributions to bodily representations. Cogn Neuropsychol 33:67-81.0.1080/02643294.2016.1168390.

Ferrè ER, Berlot E, and Haggard P. 2015. Vestibular contributions to a right-hemisphere network for bodily awareness: combining galvanic vestibular stimulation and the "Rubber Hand Illusion". Neuropsychologia 69:140-147.

Ferrè ER, Bottini G, and Haggard P. 2011. Vestibular modulation of somatosensory perception. Eur J Neurosci 34:1337-1344.

Ferrè ER, Bottini G, Iannetti GD, and Haggard P. 2013. The balance of feelings: vestibular modulation of bodily sensations. Cortex 49:748-758. 10.1016/j.cortex.2012.01.012.

Ferrè ER, Haggard P, Bottini G, and Iannetti GD. 2015. Caloric vestibular stimulation modulates nociceptive evoked potentials. Exp Brain Res 233:3393-3401. 10.1007/s00221-015-44128.

Peer) reviewing PDF | (2018:12:33926:1:2:NEW 10 May 2019) 
589 Gallace A, and Bellan V. 2018. The parietal cortex and pain perception: a body protection

590

591

592

593

594

595

596

597

598

599

600

601

602

603

604

605

606

607

608

609

610

611

612

613

614

615

616

617

618

619

620

621

622

623 system. Handb Clin Neurol 151:103-117.

Gallace A, Soravia G, Cattaneo Z, Moseley GL, and Vallar G. 2014. Temporary interference over the posterior parietal cortices disrupts thermoregulatory control in humans. PLoS One 9:e88209. 10.1371/journal.pone.0088209.

Grabherr L, Cuffel C, Guyot J-P, and Mast FW. 2011. Mental transformation abilities in patients with unilateral and bilateral vestibular loss. Exp Brain Res 209:205-214.

Grabherr L, Macauda G, and Lenggenhager B. 2015. The moving history of vestibular stimulation as a therapeutic intervention. Multisens Res 28:653-687.

Ionta S, Heydrich L, Lenggenhager B, Mouthon M, Fornari E, Chapuis D, Gassert R, and Blanke O. 2011. Multisensory mechanisms in temporo-parietal cortex support self-location and first-person perspective. Neuron 70:363-374. 10.1016/j.neuron.2011.03.009.

JASP Team (2018). JASP (Version 0.8.6)[Computer software].

Kammers MP, Rose K, and Haggard P. 2011. Feeling numb: temperature, but not thermal pain, modulates feeling of body ownership. Neuropsychologia 49:1316-1321. 10.1016/j.neuropsychologia.2011.02.039.

Karnath HO, Himmelbach M, and Perenin MT. 2003. Neglect-like behaviour in healthy subjects: dissociation of space exploration and goal-directed pointing after vestibular stimulation. Exp Brain Res 153:231-238.

Lee H, Lamb SE, Bagg MK, Toomey E, Cashin AG, and Moseley GL. 2018. Reproducible and replicable pain research: a critical review. Pain 159:1683-1689. 10.1097/j.pain/0000000000001254.

Lenggenhager B, and Lopez C. 2016. Vestibular contributions to the sense of body, self, and others. Frankfurt am Main: MIND Group.

Lenggenhager B, Hilti L, Palla A, Macauda G, and Brugger P. 2014. Vestibular stimulation does not diminish the desire for amputation. Cortex 54:210-212.

Lenggenhager B, Lopez C, and Blanke O. 2008. Influence of galvanic vestibular stimulation on egocentric and object-based mental transformations. Exp Brain Res 184:211-221.

Lopez C, and Elzière M. 2018. Out-of-body experience in vestibular disorders-A prospective study of 210 patients with dizziness. Cortex 104:193-206.

Lopez C, Nakul E, Preuss N, Elzière M, and Mast FW. 2018. Distorted own-body representations in patients with dizziness and during caloric vestibular stimulation. $J$ Neurol:1-9.

Lopez C. 2015. Making sense of the body: The role of vestibular signals. Multisens Res 28:525557. 
624 Lopez C, Bieri CP, Preuss N, and Mast FW. 2012. Tactile and vestibular mechanisms underlying

625

626

627

628

629

630

631

632

633

634

635

636

637

638

639

640

641

642

643

644

645

646

647

648

649

650

651

652

653

654

655

656

657

658 ownership for body parts: a non-visual variant of the rubber hand illusion. Neurosci Lett 511:120-124.

Lopez C, Blanke O, and Mast F. 2012. The human vestibular cortex revealed by coordinatebased activation likelihood estimation meta-analysis. Neuroscience 212:159-179.

Lopez C, Lenggenhager B, and Blanke O. 2010. How vestibular stimulation interacts with illusory hand ownership. Conscious Cogn 19:33-47.

Lopez C, Schreyer HM, Preuss N, and Mast FW. 2012. Vestibular stimulation modifies the body schema. Neuropsychologia 50:1830-1837. 0.1016/j.neuropsychologia.2012.04.008.

Macauda G, Bertolini G, Palla A, Straumann D, Brugger P, and Lenggenhager B. 2015. Binding body and self in visuo-vestibular conflicts. Eur J Neurosci 41(6):810-7. 10.1111/ejn.12809.

Macrea LM, Macauda G, Bertolini G, Straumann D, Brugger P, Maurer K, Palla A, and Lenggenhager B. 2016. Reducing pain by moving? A commentary on Ferrè et al. 2013. Cortex 78:167-169.

Mast FW, Preuss N, Hartmann M, and Grabherr L. 2014. Spatial cognition, body representation and affective processes: the role of vestibular information beyond ocular reflexes and control of posture. Front Integr Neurosci 8:44.

Miller SM. 2016. Vestibular neuromodulation: stimulating the neural crossroads of psychiatric illness. Bipolar disorders 18:539-543.

Miller SM, and Ngo TT. 2007. Studies of caloric vestibular stimulation: implications for the cognitive neurosciences, the clinical neurosciences and neurophilosophy. Acta Neuropsychiatr 19:183-203.

Moseley GL, Gallace A, and Iannetti GD. 2012. Spatially defined modulation of skin temperature and hand ownership of both hands in patients with unilateral complex regional pain syndrome. Brain 135:3676-3686. 10.1093/brain/aws297.

Moseley GL, Olthof N, Venema A, Don S, Wijers M, Gallace A, and Spence C. 2008. Psychologically induced cooling of a specific body part caused by the illusory ownership of an artificial counterpart. Proc Natl Acad Sci US A 105:13169-13173. 10.1073/pnas.0803768105.

Moseley GL, Gallace A, and Spence C. 2012. Bodily illusions in health and disease: physiological and clinical perspectives and the concept of a cortical 'body matrix'. Neurosci Biobehav Rev 36:34-46. 10.1016/j.neubiorev.2011.03.013.

Newport R, and Gilpin HR. 2011. Multisensory disintegration and the disappearing hand trick. Curr Bio 21:R804-R805. 
659

660

661

662

663

664

665

666

667

668

669

670

671

672

673

674

675

676

677

678

679

680

681

682

683

684

685

686

687

688

689

690

691

692

693

694

Ngo TT, Liu GB, Tilley AJ, Pettigrew JD, and Miller SM. 2007. Caloric vestibular stimulation reveals discrete neural mechanisms for coherence rivalry and eye rivalry: a meta-rivalry model. Vision Res 47:2685-2699. 10.1016/j.visres.2007.03.024.

Ngo TT, Liu GB, Tilley AJ, Pettigrew JD, and Miller SM. 2008. The changing face of perceptual rivalry. Brain Res Bull 75:610-618. 10.1016/j.brainresbull.2007.10.006.

Palla A, and Lenggenhager B. 2014. Ways to investigate vestibular contributions to cognitive processes. Front Integr Neurosci 8:40.

Pfeiffer C, Lopez C, Schmutz V, Duenas JA, Martuzzi R, and Blanke O. 2013. Multisensory origin of the subjective first-person perspective: visual, tactile, and vestibular mechanisms. PLoS One 8:e61751.

Pfeiffer C, Van Elk M, Bernasconi F, and Blanke O. 2016. Distinct vestibular effects on early and late somatosensory cortical processing in humans. Neuroimage 125:208-219.

Ponzo S, Kirsch LP, Fotopoulou A, and Jenkinson PM. 2018. Balancing body ownership: Visual capture of proprioception and affectivity during vestibular stimulation. Neuropsychologia 117:311-321. 10.1016/j.neuropsychologia.2018.06.020.

Preuss N, and Ehrsson HH. 2018. Full-body ownership illusion elicited by visuo-vestibular integration. J Exp Psychol Hum Percept Perform 10.1037/xhp0000597.

Price DD, Staud R, and Robinson ME. 2012. How should we use the visual analogue scale (VAS) in rehabilitation outcomes? II: Visual analogue scales as ratio scales: an alternative to the view of Kersten et al. J Rehab Med 44(9):800-1 . 10.2340/165019771031.

Reid EJ, Braithwaite FA, Wallwork SB, Harvie D, Chalmers KJ, Spence C, Gallace A, and Moseley GL. 2018. Spatially-defined motor deficits in people with unilateral complex regional pain syndrome. Cortex 104:154-162.

Reid E, Wallwork SB, Harvie D, Chalmers KJ, Gallace A, Spence C, and Moseley GL. 2016. A new kind of spatial inattention associated with chronic limb pain? Ann Neurol 79:701704. 10.1002/ana.24616.

Rohde M, Di Luca M, and Ernst MO. 2011. The Rubber Hand Illusion: Feeling of ownership and proprioceptive drift do not go hand in hand. PLoS One 6:e21659. 10.1371/journal.pone.0021659.

Schmidt L, Artinger F, Stumpf O, and Kerkhoff G. 2013. Differential effects of galvanic vestibular stimulation on arm position sense in right- vs. left-handers. Neuropsychologia 51:893-899. 10.1016/j.neuropsychologia.2013.02.013.

Schmidt L, Keller I, Utz KS, Artinger F, Stumpf O, and Kerkhoff G. 2013. Galvanic vestibular stimulation improves arm position sense in spatial neglect: a sham-stimulation-controlled study. Neurorehabil Neural Repair 27:497-506.

Peer] reviewing PDF | (2018:12:33926:1:2:NEW 10 May 2019) 
695 Serino A, Alsmith A, Costantini M, Mandrigin A, Tajadura-Jimenez A, and Lopez C. 2013.

696

697

698

699

700

701

702

703

704

705

706

707

708 Bodily ownership and self-location: components of bodily self-consciousness. Conscious Cogn 22:1239-1252. 10.1016/j.concog.2013.08.013.

Tsakiris M, and Haggard P. 2005. The Rubber Hand Illusion revisited: Visuotactile integration and self-attribuation. J Exp Psychol Hum Percept Perform 31:80-91.

Veale JF. 2014. Edinburgh handedness inventory-short form: a revised version based on confirmatory factor analysis. Laterality 19:164-177.

Wallwork SB, Bellan V, Catley MJ, and Moseley GL. 2015. Neural representations and the cortical body matrix: implications for sports medicine and future directions. Br J Sports Med. 10.1136/bjsports-2015-095356.

Wallwork SB, Butler DS, and Moseley GL. 2013. Dizzy people perform no worse at a motor imagery task requiring whole body mental rotation; a case-control comparison. Front Hum Neurosci 7:258. 


\section{Table $\mathbf{1}$ (on next page)}

Analysis of MIRAGE questions.

${ }^{\S}$ Friedman Two-Way ANOVA by Rank; ${ }^{\dagger}$ Wilcoxon Signed Rank Test performed on the left (L) and right (R) hands separately; 'Substituted the word 'left' for 'right' when the left hand was tested; *significant $p<.05,{ }^{*}$ significant $p<.025$. 
1 Table 1 Analysis of MIRAGE questions

\begin{tabular}{|c|c|c|}
\hline Questions & Friedman $^{\S}$ & post hoc Wilcoxon ${ }^{\dagger}$ \\
\hline \multicolumn{3}{|c|}{ Self-location } \\
\hline 1. I knew exactly where my right ${ }^{\star}$ hand was. & $X^{2}=2.27, p=.519$ & \\
\hline 5. I couldn't tell where my right" hand was. & $X^{2}=5.05, p=.168$ & \\
\hline \multicolumn{3}{|c|}{ Sense of ownership } \\
\hline 2. My right" hand was part of my body. & $X^{2}=2.61, p=.456$ & \\
\hline $\begin{array}{l}\text { 7. It seemed that my right } \$ \text { hand no longer } \\
\text { belonged to me. }\end{array}$ & $X^{2}=3.12, p=.372$ & \\
\hline \multicolumn{3}{|c|}{ Self-location and sense of ownership } \\
\hline $\begin{array}{l}\text { 4. It seemed like my right }{ }^{\ddagger} \text { hand was no longer } \\
\text { there. }\end{array}$ & $X^{2}=8.52, p=.036^{*}$ & $\begin{array}{l}\mathrm{L}: \mathrm{Z}=-2.50, p=.012^{\#} \\
\mathrm{R}: \mathrm{Z}=-2.06, p=.039\end{array}$ \\
\hline \multicolumn{3}{|c|}{ Sensation of the hand } \\
\hline $\begin{array}{l}\text { 3. The sensation in my right* hand was more } \\
\text { vivid than normal. }\end{array}$ & $X^{2}=1.79, p=.688$ & \\
\hline $\begin{array}{l}\text { 6. I had the sensation that my right } * \text { hand was } \\
\text { numb. }\end{array}$ & $X^{2}=7.23, p=.065$ & \\
\hline
\end{tabular}

2

3 §Friedman Two-Way ANOVA by Rank; ${ }^{\dagger}$ Wilcoxon Signed Rank Test performed on the left (L) 4 and right ( $\mathrm{R}$ ) hands separately; $\$$ Substituted the word 'left' for 'right' when the left hand was 5 tested; * significant $p<.05$, ${ }^{*}$ significant $p<.025$. 


\section{Figure 1}

Disappearing hand procedure using the MIRAGE multisensory illusion box.

Depicted is the participant's perspective while watching her hands during the adaptation procedure inside the MIRAGE box at the beginning of the procedure $(A)$ and towards the end (B). The actual hand end position is further outwards compared to the seen position. Please note that these pictures are only for illustrating purposes, the participants did not actually see their elbows, which were occluded from view with a bib to prevent visual cues about the actual hand position. As indicated during the debriefing, most participants were not aware of the incongruence between the visual and physical hand position. Photo credit: Cat Jones.

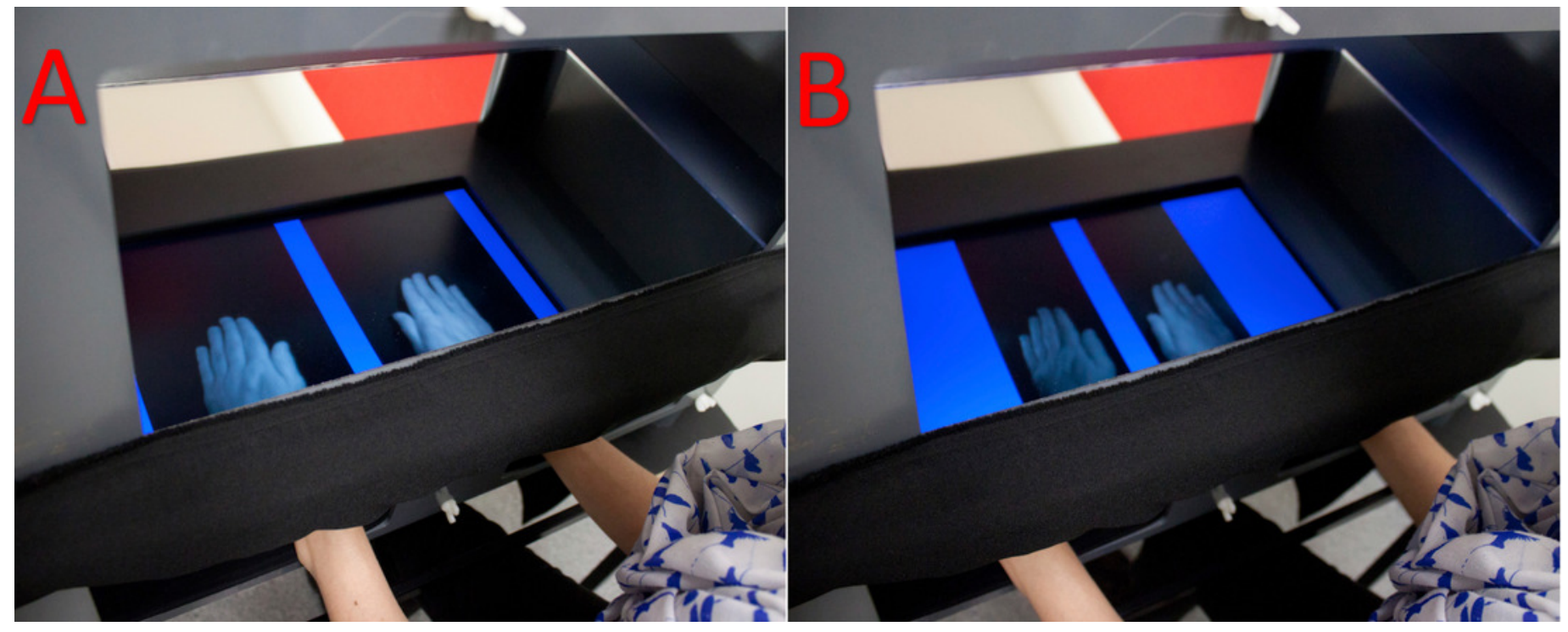


Figure 2

Average drift.

Localisation of the middle finger, average for all participants at each of the 6 trials; standard deviation bars shown going up for CVS and down for sham. Slope and intercept were calculated based on these results. Data markers for left and right hands are shifted slightly left and right, respectively, of trial number to more clearly differentiate the data. Distance is measured from $0=$ visual location to $13.65 \mathrm{~cm}=$ physical location of the middle finger.

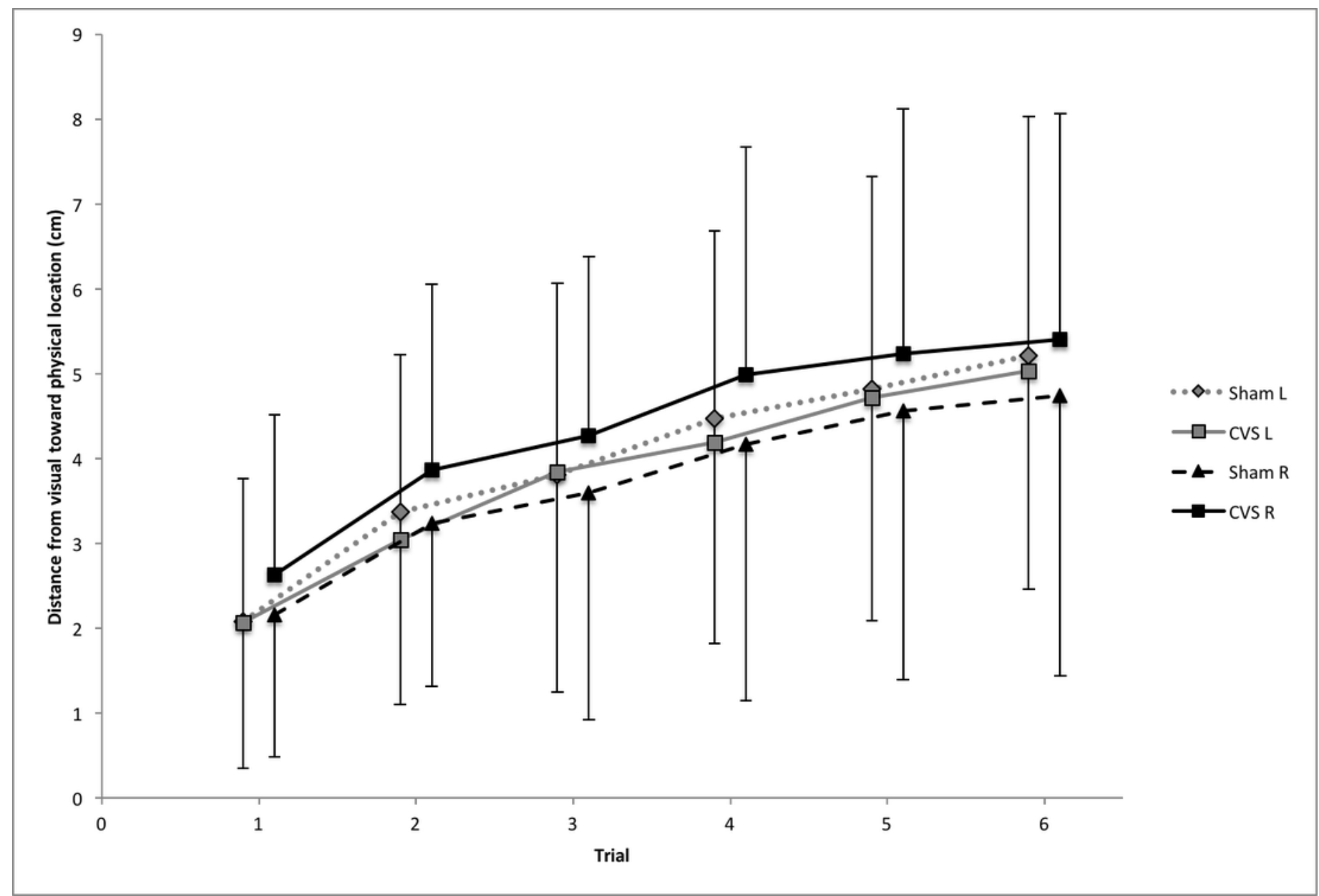

